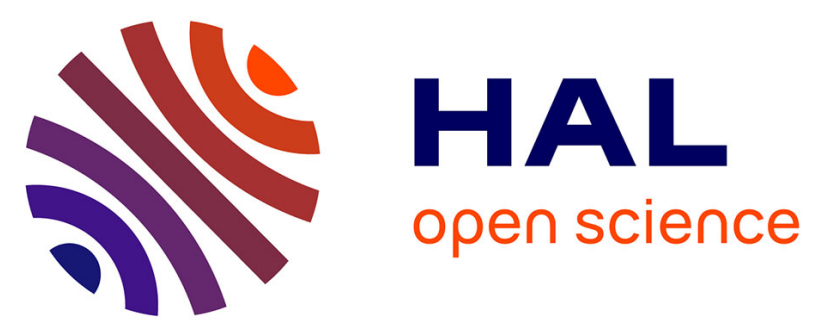

\title{
High-quality construction of analysis-suitable trivariate NURBS solids by reparameterization methods
}

\author{
Gang Xu, Bernard Mourrain, André Galligo, Timon Rabczuk
}

\section{To cite this version:}

Gang Xu, Bernard Mourrain, André Galligo, Timon Rabczuk. High-quality construction of analysissuitable trivariate NURBS solids by reparameterization methods. Computational Mechanics, 2014, 54 (5), pp.1303-1313. 10.1007/s00466-014-1060-y · hal-00922544v2

\section{HAL Id: hal-00922544 \\ https://hal.science/hal-00922544v2}

Submitted on 8 Jan 2015

HAL is a multi-disciplinary open access archive for the deposit and dissemination of scientific research documents, whether they are published or not. The documents may come from teaching and research institutions in France or abroad, or from public or private research centers.
L'archive ouverte pluridisciplinaire HAL, est destinée au dépôt et à la diffusion de documents scientifiques de niveau recherche, publiés ou non, émanant des établissements d'enseignement et de recherche français ou étrangers, des laboratoires publics ou privés. 
Noname manuscript No.

(will be inserted by the editor)

\title{
High-quality construction of analysis-suitable trivariate NURBS solids by reparameterization methods
}

\author{
Gang Xu • Bernard Mourrain • André Galligo - Timon Rabczuk
}

Received: date / Accepted: date

\begin{abstract}
High-quality volumetric parameterization of computational domain plays an important role in threedimensional isogeometric analysis. Reparameterization technique can improve the distribution of isoparametric curves/surfaces without changing the geometry. In this paper, using the reparameterization method, we investigate the high-quality construction of analysis-suitable NURBS volumetric parameterization. Firstly, we introduce the concept of volumetric reparameterization, and propose an optimal Möbius transformation to improve the quality of the isoparametric structure based on a new uniformity metric. Secondly, from given boundary NURBS surfaces, we present a two-stage scheme to construct the analysis-suitable volumetric parameterization: in the first step, uniformity-improved reparameterization is performed on the boundary surfaces to achieve high-quality isoparametric structure without changing the shape; in the second step, from a new variational harmonic metric and the reparameterized boundary surfaces, we construct the optimal inner control points and weights to achieve an analysis-suitable
\end{abstract}

G. Xu

College of Computer Science, Hangzhou Dianzi University, Hangzhou 310018, P.R.China

E-mail: xugangzju@gmail.com

B. Mourrain

Galaad, INRIA Sophia-Antipolis, 2004 Route des Lucioles, 06902 Cedex, France

E-mail: Bernard.Mourrain@inria.fr

A. Galligo

University of Nice Sophia-Antipolis, 06108 Nice Cedex 02, France

E-mail: galligo@unice.fr

T. Rabczuk

Institute of Structural Mechanics, Bauhaus-University Weimar, Marienstr. 15, D-99423 Weimar, Germany

E-mail: timon.rabczuk@uni-weimar.de
NURBS solid. Several examples with complicated geometry are presented to illustrate the effectiveness of proposed methods.

Keywords Isogeometric analysis · Volumetric parameterization · Boundary reparameterization · Uniformity metric

\section{Introduction}

The isogeometric analysis method proposed by Hughes et al. [14] employs the same type of spline representation both for the geometry and for the physical solutions. This unified data representation allows for a seamless integration of the geometric design in numerical analysis. Moreover, the higher-order continuity of the (spline) basis function have advantageous over traditional $C^{0}$ finite element formulations based on Lagrange polynomials which can be exploited in thin shell analysis [19] or weakly non-local continuum models. The reduced number of parameters needed to describe the geometry is also of particular interest for shape and topology optimization.

Since isogeometric analysis was firstly proposed by Hughes et al. [14] in 2005, many researchers working on computational mechanical and geometric modeling were involved in this field. We can classify the current work on isogeometric analysis into four categories: (1) isogeometric application in multi-physical problems [3, 9,13]; (2) application of different spline models in isogeometric analysis $[4,5,10,21,15]$; (3) improving the accuracy and efficiency of IGA framework by refinement operations and parallel computing $[2,6,8,28,29]$; (4) constructing analysis-suitable parameterization of computational domain from given boundary $[1,18,20,26,29$, $30,33,34]$. 
The work presented this paper belongs to the fourth category. Mesh generation from a given CAD object is one of the most time-consuming step in a numerical analysis based on finite elements [14]. In the isogeometric analysis framework, the parameterization of the computational domain corresponds to the mesh generation in finite element analysis and is the key of an effective isogeometric analysis that was developed for the main purpose of drastically shortening the modeling time in numerical analysis.

Constructing analysis-suitable parameterization from a given CAD boundary representation remains one of the most significant challenges in isogeometric analysis. In $[6,28]$, the authors study the parametrization of computational domain in IGA, and show that the quality of parameterization has great impact on analysis results and efficiency. Pilgerstorfer and Jüttler show that in isogeometric analysis the condition number of the stiffness matrix, which is a key factor for the stability of the linear system, depends strongly on the quality of domain parameterization [23]. Using volumetric harmonic functions, Martin et al. [18] proposed a fitting method for triangular mesh by B-spline parametric volumes. Aigner et al. [1] proposed a variational approach to construct NURBS parameterization of swept volumes. In [12], a method is proposed to construct trivariate Tspline volumetric parameterization for genus-zero solid based on an adaptive tetrahedral meshing and mesh untangling technique. Zhang et al. proposed a robust and efficient approach to construct injective solid T-splines for genus-zero geometry from a boundary triangulation [33]. For mesh model with arbitrary topology, volumetric parameterization methods are proposed from the Morse theory [26] and Boolean operations [17].The input data of above methods is a triangle mesh. For the product modeling by CAD software, its boundary is usually in spline form. For parameterization problem with spline boundary, $\mathrm{Xu}$ et al. proposed a constraint optimization framework to construct analysissuitable volume parameterization [30]. Spline volume faring is proposed by Pettersen and Skytt to obtain high-quality volume parameterization for isogeometric applications [24]. The construction of conformal solid $\mathrm{T}$-spline from boundary T-spline representation is studied by using octree structure and boundary offset [34]. In [31], variational harmonic method is proposed to construct analysis-suitable parameterization of computational domain from given CAD boundary information. Wang and Qian proposed an efficient method by combining divide-and-conquer, constraint aggregation and the hierarchical optimization technique to obtain valid trivariate B-spline solids from six boundary B-spline surfaces [27].
An analysis-suitable parameterization of computational domain in isogeometric analysis should satisfy three requirements: 1) it should have no self-intersections, i.e, the mapping from the parametric domain to physical domain should be injective; 2) the iso-parametric elements should be as uniform as possible; 3 ) the isoparametric structure should be as orthogonal as possible. Previous work mainly focus on the construction of inner control points $[33,34,30,31,27]$. To our best knowledge, the effect of the boundary parameterization on the interior volumetric parameterization has not been studied before. However, the quality of boundary parameterization has great effect on the subsequent volumetric parameterization results. Reparameterization technique can improve the quality of boundary parameterization without changing the geometry. On the other hand, the weights in NURBS solid can be also considered as extra degree of freedom to obtain highquality volumetric parameterization. In this paper, using the reparameterization method, we investigate the high-quality construction of analysis-suitable NURBS volumetric parameterization. Our main contributions are:

- Two kinds of new volumetric metrics are introduced. A uniformity metric is developed from the geometric interpretation of the Jacobian and the concept of variance in statistics science; a new harmonic metric for volumetric smoothing is introduced from the variational formulation of harmonic equation.

- Volumetric spline reparameterization is introduced, and an optimal Möbius transformation is proposed to improve the uniformity of isoparametric structure.

- By using uniformity-improved reparameterization of NURBS surfaces and the proposed variational harmonic metric, a two-stage scheme with multi-objective functions is proposed to construct the optimal inner control points and weights for analysis-suitable NURBS volumetric parameterization.

The rest of the paper is structured as follows. After a new uniformity metric is introduced, Section 2 describes the optimal Mobius volumetric reparameterization method for analysis-suitable NURBS solids. For volumetric parameterization problem from given boundaries, a two-stage framework with multi-objective function is proposed to construct the optimal analysis-suitable NURBS solid in Section 3. Some examples and comparisons are also presented in corresponding sections to illustrate the effectiveness of the proposed methods. Finally, we conclude this paper and outline future works in Section 4. 


\section{Volumetric reparameterization for analysis-suitable NURBS solids}

\subsection{Problem statement}

The problem studied in this section can be stated as follows: given a trivariate NURBS solid, find an optimal Möbius parameter transformation such that the isoparametric structure of reparameterized NURBS solid is as uniform as possible.

In the following subsection, we will review the definition of NURBS solids and introduce the Mobius volumetric transformation.

2.2 NURBS solids and Mobius volumetric transformation

A NURBS solid can be defined as follows,

$\mathbf{S}(u, v, w)=\frac{\sum_{i=0}^{n} \sum_{j=0}^{m} \sum_{k=0}^{l} \lambda_{i, j, k} \boldsymbol{C}_{i, j, k} N_{i}^{p}(u) N_{j}^{q}(v) N_{k}^{\nu}(w)}{\sum_{i=0}^{n} \sum_{j=0}^{m} \sum_{k=0}^{l} \lambda_{i, j, k} N_{i}^{p}(u) N_{j}^{q}(v) N_{k}^{\nu}(w)}$

in which $\boldsymbol{C}_{i, j, k}$ are control points, $\lambda_{i, j, k}$ are the weights, $N_{i}^{p}(u), N_{j}^{q}(v)$ and $N_{k}^{\nu}(w)$ are B-spline basis function with degree $p, q$ and $\nu$ respectively defined on the knot vectors

$\mathbf{U}=\left\{0, \cdots, 0, u_{p+1}, \cdots, u_{l}, 1, \cdots, 1\right\}$

$\mathbf{V}=\left\{0, \cdots, 0, v_{q+1}, \cdots, v_{m}, 1, \cdots, 1\right\}$

and

$\mathbf{W}=\left\{0, \cdots, 0, w_{r+1}, \cdots, w_{n}, 1, \cdots, 1\right\}$

Definition 1 (Mobius volumetric transformation) Suppose that $\alpha, \beta, \gamma \in[0,1]$, the Mobius volumetric transformation can be defined as

$u=\frac{(1-\alpha) \xi}{\alpha(1-\xi)+(1-\alpha) \xi}$

$v=\frac{(1-\beta) \eta}{\beta(1-\eta)+(1-\beta) \eta}$

$w=\frac{(1-\gamma) \zeta}{\gamma(1-\zeta)+(1-\gamma) \zeta}$

After applying the Mobius transformation in $(1)(2)(3)$ on the NURBS solid $\mathbf{S}(u, v, w)$, we can obtain a new parametric representation $\widetilde{\mathbf{S}}(\xi, \eta, \zeta)$ of the NURBS solid with the same control points as follows [16],

$$
\begin{aligned}
\widetilde{\mathbf{S}}(\xi, \eta, \zeta) & =(x(\xi, \eta, \zeta), y(\xi, \eta, \zeta), z(\xi, \eta, \zeta)) \\
& =\frac{\sum_{i=0}^{n} \sum_{j=0}^{m} \sum_{k=0}^{l} \widetilde{\lambda}_{i, j, k} \boldsymbol{C}_{i, j, k} N_{i}^{p}(\xi) N_{j}^{q}(\eta) N_{k}^{\nu}(\zeta)}{\sum_{i=0}^{n} \sum_{j=0}^{m} \sum_{k=0}^{l} \widetilde{\lambda}_{i, j, k} N_{i}^{p}(\xi) N_{j}^{q}(\eta) N_{k}^{\nu}(\zeta)}
\end{aligned}
$$

in which the new weights

$\tilde{\lambda}_{i, j, k}=\frac{\lambda_{i, j, k}}{\prod_{r=1}^{p} K_{i, r} \prod_{s=1}^{q} L_{j, s} \prod_{t=1}^{\nu} M_{k, t}}$

with

$K_{i, r}=(1-\alpha)\left(1-u_{i+r}\right)+\alpha u_{i+r}$,

$L_{j, s}=(1-\beta)\left(1-v_{j+s}\right)+\beta v_{j+s}$,

$M_{k, t}=(1-\gamma)\left(1-w_{k+t}\right)+\gamma w_{k+t}$.

And the corresponding knot vectors are changed into

$$
\begin{aligned}
\widetilde{\mathbf{U}}= & \{\underbrace{0, \cdots, 0}_{p+1}, \frac{\alpha u_{p+1}}{(1-\alpha)\left(1-u_{p+1}\right)+\alpha u_{p+1}}, \cdots, \\
& \frac{\alpha u_{l}}{(1-\alpha)\left(1-u_{l}\right)+\alpha u_{l}}, \underbrace{1, \cdots, 1}_{p+1}\} \\
\widetilde{\mathbf{V}=} & \{\underbrace{0, \cdots, 0}_{q+1}, \frac{\beta v_{q+1}}{(1-\beta)\left(1-v_{q+1}\right)+\beta v_{q+1}}, \cdots, \\
& \frac{\beta v_{m}}{(1-\beta)\left(1-v_{m}\right)+\beta v_{m}}, \underbrace{1, \cdots, 1}_{q+1}\}
\end{aligned}
$$

and

$$
\begin{aligned}
\widetilde{\mathbf{W}}= & \{\underbrace{0, \cdots, 0}_{\nu+1}, \frac{\gamma w_{\nu+1}}{(1-\gamma)\left(1-w_{\nu+1}\right)+\gamma w_{\nu+1}}, \cdots, \\
& \frac{\gamma w_{n}}{(1-\gamma)\left(1-w_{n}\right)+\gamma w_{n}}, \underbrace{1, \cdots, 1}_{\nu+1}\} .
\end{aligned}
$$

2.3 Improving the uniformity of isoparametric structure by volumetric reparameterization

In this subsection, we will propose a uniformity-improved volumetric reparameterization method based a new uniformity metric. 


\subsubsection{Uniformity metric for NURBS solids}

In order to achieve a volumetric parameterization with uniform isoparametric structure, a new uniformity metric is firstly proposed. The uniform isoparametric structure means that each isoparametric element has the same volume value. In probability theory and statistics, variance measures how far a set of numbers is spread out. A variance of zero indicates that all the values are identical. Hence, the uniformity of isoparametric structure means that the variane between the volume value of each isoparametric element should be as small as possible. Suppose that $V_{i}$ is the volume of $i$-th element, and $V_{a v e}$ is the average element volume in the NURBS solid $\widetilde{\boldsymbol{S}}(\xi, \eta, \zeta)$, the discrete variance $\sigma_{d i s}$ can be defined as

$\sigma_{d i s}=\frac{1}{N} \sum_{i=0}^{N}\left(V_{i}-V_{a v e}\right)^{2}$

in which $N$ is the number of sampling elements.

The Jacobian determinant can be considered as a scaling factor that relates the volume change of the parametric element to the physical element. Hence, the variance of element volume in Eq. (7) can be replaced by the variance of Jacobian determinant of the NURBS solid $\widetilde{\boldsymbol{S}}(\xi, \eta, \zeta)$, which can be defined in the form the continuous function as

$\sigma=\frac{\int_{\mathcal{P}}\left(\operatorname{det} \widetilde{\mathbf{J}}-J_{\text {mean }}\right)^{2} d \mathcal{P}}{\int_{\mathcal{P}} d \xi d \eta d \zeta}$

in which $J_{\text {mean }}$ is the average value of Jacobian determinant at each sampling point on the NURBS solid. $J_{\text {mean }}$ can be computed as the ratio between the volume value of physical domain $V_{\text {physical }}$ and parametric domain $V_{\text {parametric }}$

$$
\begin{aligned}
J_{\text {mean }} & =\frac{V_{\text {physical }}}{V_{\text {parametric }}} \\
= & \frac{\int_{\mathcal{P}} \widetilde{\boldsymbol{S}}_{\zeta} \cdot\left(\widetilde{\boldsymbol{S}}_{\xi} \times \widetilde{\boldsymbol{S}}_{\eta}\right) d \xi d \eta d \zeta}{\int_{\mathcal{P}} d \xi d \eta d \zeta}
\end{aligned}
$$

$=\frac{\int_{\mathcal{P}} \operatorname{det} \widetilde{\mathbf{J}} d \xi d \eta d \zeta}{\int_{\mathcal{P}} d \xi d \eta d \zeta}$

in which $\mathcal{P}$ is the parametric domain with knot vectors $\widetilde{\mathbf{U}}, \widetilde{\mathbf{V}}$ and $\widetilde{\mathbf{W}} . \widetilde{\mathbf{J}}$ is the Jacobian matrix of the NURBS solid $\widetilde{\boldsymbol{S}}(\xi, \eta, \zeta)$ as follows,

$\widetilde{\mathbf{J}}=\left(\begin{array}{lll}x_{\xi} & x_{\eta} & x_{\zeta} \\ y_{\xi} & y_{\eta} & y_{\zeta} \\ z_{\xi} & z_{\eta} & z_{\zeta}\end{array}\right)$

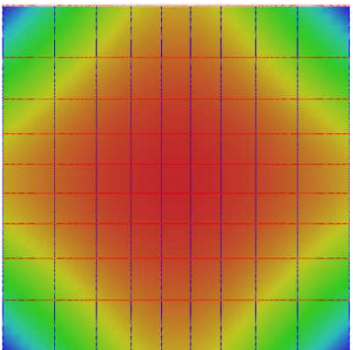

Fig. 1 The uniformity metric illustrated by color-map.

As shown in $[27,30]$, the uniformity is also related to the second order derivative of the parameterization. By combining the variance of Jacobian in (8), a new uniformity metric at $(\xi, \eta, \zeta)$ can be defined as follows,

$\mu(\widetilde{\boldsymbol{S}})=\left(\operatorname{det} \widetilde{\mathbf{J}}-J_{\text {mean }}\right)^{2}+\omega\left(\left\|\widetilde{\boldsymbol{S}}_{\xi \xi}\right\|^{2}+\left\|\widetilde{\boldsymbol{S}}_{\eta \eta}\right\|^{2}+\left\|\widetilde{\boldsymbol{S}}_{\zeta \zeta}\right\|^{2}\right)$,

in which $\omega$ is a positive weight.

In order to show the effectiveness of the proposed metric, we present an example in Figure 1. The uniformity metric is illustrated with color-map, which is rendered according to the value of $\mu(\widetilde{\boldsymbol{S}})$. The red part has smallest value and the best uniformity, the blue part has the biggest value and the worst uniformity. We can find that the uniformity color-map is consistent with the size change of the isoparametric element.

\subsubsection{Optimal volumetric Mobius reparameterization}

From the volumetric Möbius transformation and uniformity metric presented in previous sections, the optimal volumetric Möbius reparameterization problem can be stated as: given the initial parameterization $\mathbf{S}(u, v, w)$ of NURBS solid, find the optimal $\alpha, \beta, \gamma$ in the Möbius volumetric transformation $(4)(5)(6)$, such that the resulted parameterization $\widetilde{\boldsymbol{S}}(\xi, \eta, \zeta)$ minimizes the following objective function

$F_{\text {unif }}(\alpha, \beta, \gamma)=\int_{\mathcal{P}} \mu(\widetilde{\boldsymbol{S}}) d \mathcal{P}$

in which $\mu(\widetilde{\boldsymbol{S}})$ is defined in (10).

We solve this non-linear optimization problem with the Levenberg-Marquardt method to obtain the values of $\alpha, \beta$ and $\gamma$. The algorithm combines advantages of the steepest descent method, in which minimization is performed along the direction of the gradient, with the Newton method, in which a quadratic model is used to speed up the process of finding the minimum of a function. Hence, this algorithm obtained its operating stability from the steepest descent method, and adopted its accelerated convergence in the minimum vicinity from the Newton method. 


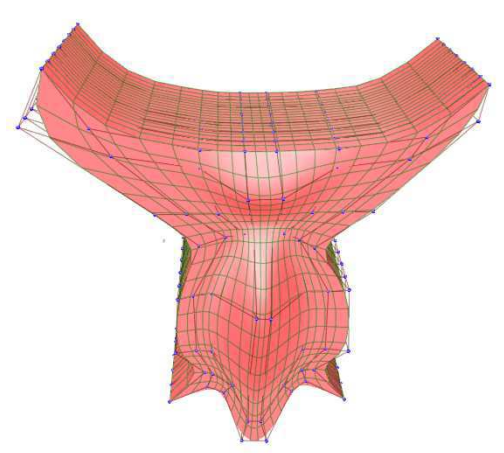

(a) NURBS solid

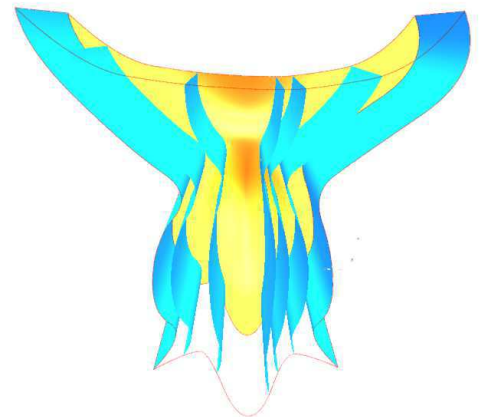

(c) Initial isoparametric surfaces in $u$ direction

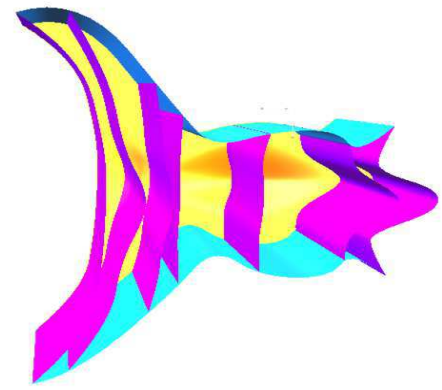

(e) Initial isoparametric surfaces in $v$ direction

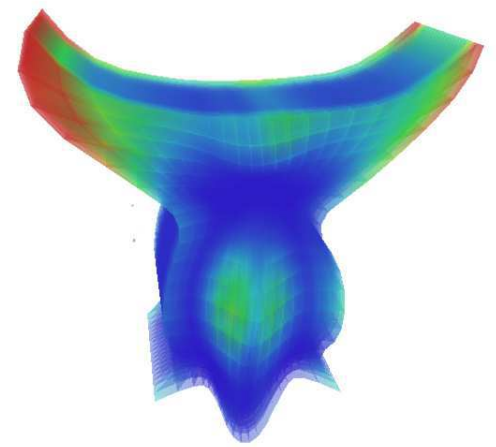

(g) Initial color-map of uniform

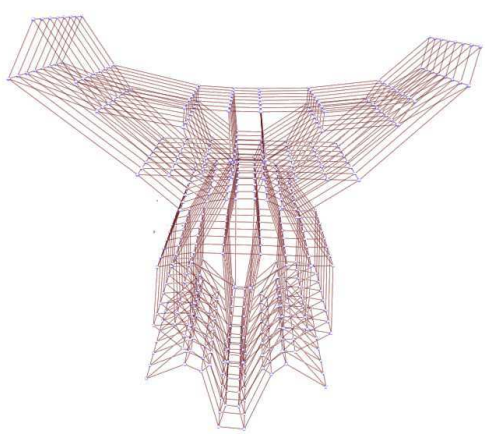

(b) Control lattice

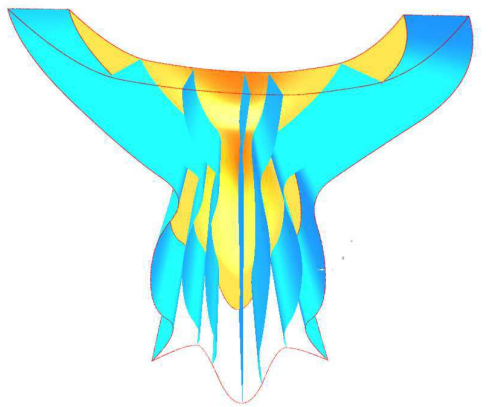

(d) Final isoparametric surfaces in $\xi$ direction

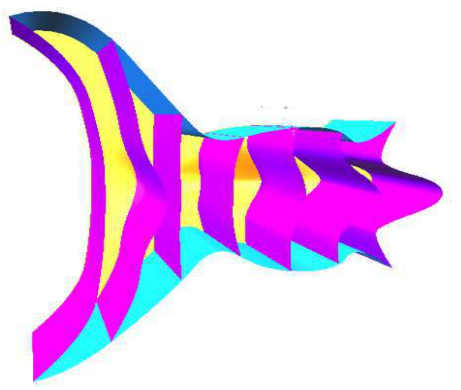

(f) Final isoparametric surfaces in $\eta$ direction

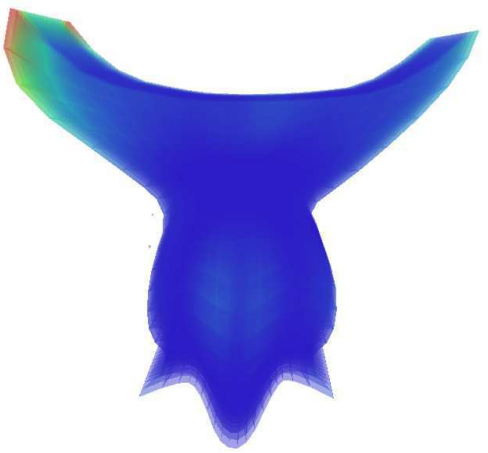

(h) Final color-map of uniform metric

Fig. 2 Volumetric Möbius reparameterization method for NURBS solid. 


\subsection{Example and comparison}

Figure 2 shows an example and corresponding comparison result for volumetric Möbius reparameterization. The given NURBS solid and the control lattice are shown in Figure 2 (a) and 2 (b). Figure 2 (c) presents the initial isoparametric surfaces in $u$ direction of the given NURBS volumetric parametrization. Figure 2 (d) shows the final isoparametric surfaces in $\xi$ direction of the NURBS volumetric parametrization constructed by the Möbius reparameterization method with $\alpha=$ $0.323, \beta=0.494$ and $\gamma=0.217$. The comparison of the isoparametric surfaces in $v$ direction are also presented in Figure 2 (e) and 2 (f). We use the uniformity colormap to show the uniformity of isoparametric structure in the volume parameterizations. The uniformity colormap is computed according to the value of $\mu(\widetilde{\boldsymbol{S}})$ defined in (10). From Fig.2(g) and Fig.2 (h) with the same scale, we can find that the volumetric parameterization obtained by the optimal Möbius reparameterization method gives more uniform iso-parametric structure than the initial given volume parameterization.

From the chain rule, we can directly prove that if initial volumetric parameterization has self-intersections, then Möbius reparameterization method can not remove the self-intersections. In the following section, we will propose a two-stage scheme to construct high-quality volumetric parameterization without self-intersections by boundary reparameterization.

\section{Constructing analysis-suitable NURBS solids by boundary reparameterization}

\subsection{Main framework}

Suppose that $\boldsymbol{S}$ is a simply connected bounded domain in three dimensional space with Cartesian coordinates $(x ; y ; z)^{T}$, and is bounded by six NURBS surfaces. The volume parameterization problem of three-dimensional computational domain in isogeometric analysis can be stated as: given six boundary NURBS surfaces, find the optimal inner control points and weights such that the resulting trivariate NURBS parametric volume is a good computational domain for 3D isogeometric analysis.

The quality of boundary parameterization has great effect on the subsequent volumetric parameterization of computational domain. In this section, we will present a two-stage scheme to construct analysis-suitable NURBS solids: in the first step, boundary surface reparameterization is performed to improve the quality of the boundary isoparametric structure; in the second step, from the reparameterized boundary surfaces, we construct the optimal inner control points and weights to achieve an analysis-suitable NURBS solid.

\section{2 boundary reparameterization}

The boundary reparameterization in this part can be viewed as the degenerated case of the volumetric reparameterization in Section 2. For each given boundary NURBS surface

$\mathbf{R}(u, v)=\frac{\sum_{i=0}^{n} \sum_{j=0}^{m} \lambda_{i, j} \boldsymbol{C}_{i, j} N_{i}^{p}(u) N_{j}^{q}(v)}{\sum_{i=0}^{n} \sum_{j=0}^{m} \lambda_{i, j} N_{i}^{p}(u) N_{j}^{q}(v),}$

The following Mobius transformation is performed on $\mathbf{R}(u, v)$

$u=\frac{(1-\alpha) \xi}{\alpha(1-\xi)+(1-\alpha) \xi}$

$v=\frac{(1-\beta) \eta}{\beta(1-\eta)+(1-\beta) \eta}$

in which $\alpha, \beta \in[0,1]$. Then we can obtain a new parametric NURBS surface $\widetilde{\mathbf{R}}(\xi, \eta)$ with the same geometry as $\mathbf{R}(u, v)$. $\widetilde{\mathbf{R}}(\xi, \eta)$ has the same control points but different weights with $\mathbf{R}(u, v)$. The new weights $\widetilde{\lambda}_{i, j, k}$ can be computed from the old weights $\lambda_{i, j, k}$ as follows,

$\widetilde{\lambda}_{i, j, k}=\frac{\lambda_{i, j, k}}{\prod_{r=1}^{p} K_{i, r} \prod_{s=1}^{q} L_{j, s}}$

with $K_{i, r}$ and $L_{j, s}$ defined in (4) and (5).

Then we seek for the optimal parameter $\alpha$ and $\beta$, such that the isoparametric net of resulting NURBS surface $\widetilde{\mathbf{R}}(\xi, \eta)$ is as uniform as possible. That is, find the optimal $\alpha$ and $\beta$ to minimize the following objective function as shown in Section

$\int_{\mathcal{P}}\left(\operatorname{det} \widetilde{\mathbf{J}}-J_{a v g}\right)^{2}+\omega_{1}\left(\left\|\widetilde{\mathbf{R}}_{\xi \xi}\right\|^{2}+\left\|\widetilde{\mathbf{R}}_{\eta \eta}\right\|^{2}\right) d \xi d \eta$,

in which

$J_{a v g}=\frac{\int_{\mathcal{P}} \operatorname{det} \mathbf{J} d \xi d \eta}{\int_{\mathcal{P}} d \xi d \eta}$

and

$\widetilde{\mathbf{J}}=\left(\begin{array}{ll}x_{\xi} & x_{\eta} \\ y_{\xi} & y_{\eta}\end{array}\right)$

Similar with the trivariate case, the LevenbergMarquardt method is used to solve this nonlinear optimization problem. 


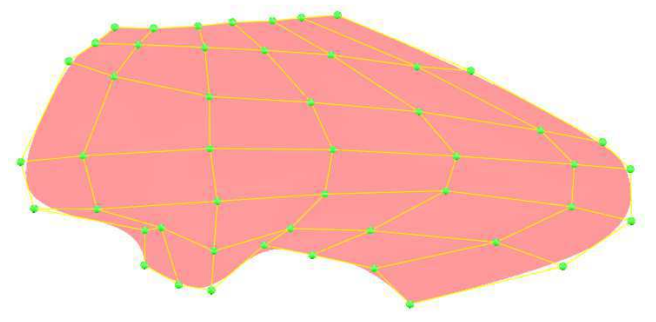

(a)

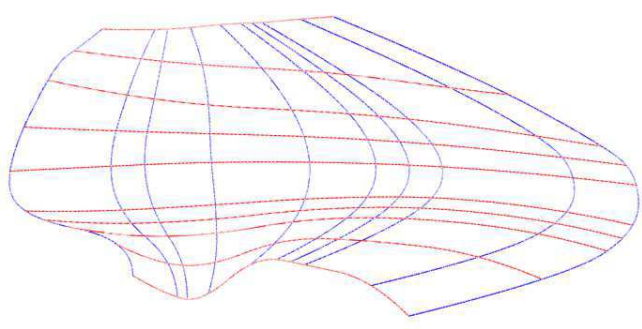

(b)

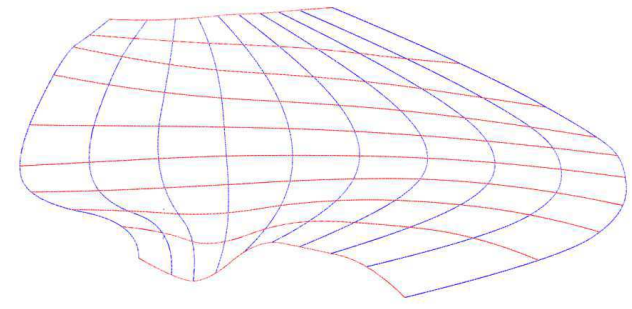

(c)

Fig. 3 Reparameterization example of a planar NURBS surfaces: (a) NURBS surface and control mesh; (b)initial isoparametric net on the surface; (c) final isoparametric net after optimal Móbius reparameterization.

Figure 3 presents an example of boundary surface reparameterization. Figure 3 (a) presents the given planar NURBS surface and its control mesh. Figure 3(b) presents the initial isoparametric net on the surface; the corresponding iso-parametric structure of the reparameterized NURBS surface obtained by optimal Möbius transformation is shown in Figure 3(c). Obviously, more uniform iso-parametric structure can be achieved without changing the boundary shape by optimal reparameterization technique.

\subsection{Initial construction of NURBS solids}

After boundary reparameterization, we need to construct the initial control points and weights for the subsequent optimization process. In [30], the discrete Coons method is proposed to construct B-spline volume by linear combination of boundary control points. In this approach, the compatible boundary surfaces with the same degree, knot vectors and the number of control points are required. However, in practice, such requirements are usually not satisfied. Hence, some pre- processing operation must be performed for the given opposite NURBS surfaces according to the following operation procedure:

- make the given opposite NURBS surfaces have the consistent parametric direction

- perform degree elevation to have the same degree

- perform knot insertion to have the same number of control points

When all the opposite surfaces on the boundary are compatible, the discrete coons method can be employed to construct the NURBS volumes [28][11] . That is, the interior control points $\boldsymbol{C}_{i, j, k}$ and weights $\lambda_{i, j, k}$ can be constructed as linear combination of boundary control points and weights. If we introduce the four-dimensional notation $\boldsymbol{P}_{i, j, k}=\left(\boldsymbol{C}_{i, j, k}, \lambda_{i, j, k}\right)$, the corresponding construction formula can be written as

$$
\begin{aligned}
& \boldsymbol{P}_{i, j, k}=(1-i / l) \boldsymbol{P}_{0, j, k}+i / l \boldsymbol{P}_{l, j, k}+(1-j / m) \boldsymbol{P}_{i, 0, k} \\
& +j / m \boldsymbol{P}_{i, m, k}+(1-k / n) \boldsymbol{P}_{i, j, 0}+k / n \boldsymbol{P}_{i, j, n} \\
& -[1-i / l, i / l]\left[\begin{array}{ll}
\boldsymbol{P}_{0,0, k} & \boldsymbol{P}_{0, m, k} \\
\boldsymbol{P}_{l, 0, k} & \boldsymbol{P}_{l, m, k}
\end{array}\right]\left[\begin{array}{c}
1-j / m \\
j / m
\end{array}\right] \\
& -[1-j / m, j / m]\left[\begin{array}{ll}
\boldsymbol{P}_{i, 0,0} & \boldsymbol{P}_{i, 0, n} \\
\boldsymbol{P}_{i, m, 0} & \boldsymbol{P}_{i, m, n}
\end{array}\right]\left[\begin{array}{c}
1-k / n \\
k / n
\end{array}\right] \\
& -[1-k / n, k / n]\left[\begin{array}{ll}
\boldsymbol{P}_{0, j, 0} & \boldsymbol{P}_{l, j, 0} \\
\boldsymbol{P}_{0, j, n} & \boldsymbol{P}_{l, j, n}
\end{array}\right]\left[\begin{array}{c}
1-i / l \\
i / l
\end{array}\right] \\
& +(1-k / n)\left[[1-i / l, i / l]\left[\begin{array}{l}
\boldsymbol{P}_{0,0,0} \\
\boldsymbol{P}_{0, m, 0}
\end{array}\right]\left[\begin{array}{c}
1-j / m \\
j / m
\end{array}\right]\right] \\
& +k / n\left[[1-i / l, i / l]\left[\begin{array}{ll}
\boldsymbol{P}_{0,0, n} & \boldsymbol{P}_{0, m, n} \\
\boldsymbol{P}_{l, 0, n} & \boldsymbol{P}_{l, m, n}
\end{array}\right]\left[\begin{array}{c}
1-j / m \\
j / m
\end{array}\right]\right]
\end{aligned}
$$

As shown in [30], the initial NURBS solid constructed by discrete Coons method may have self-intersections and low quality. In the following we will propose a method to construct the optimal inner control points and weights to achieve an analysis-suitable NURBS solid based on a new variational harmonic metric.

\subsection{Construction of analysis-suitable NURBS solids}

\subsubsection{Variational harmonic metric}

The proposed volumetric parameterization method is based on the concept of harmonic mapping, which is a one-to-one transformation for three-dimensional domains. From the harmonic mapping theory, if $f: \mathcal{S} \mapsto$ $\mathcal{P}$ is a harmonic mapping from $\mathcal{S}$ to $\mathcal{P}$, then the inverse mapping $f^{-1}: \mathcal{P} \mapsto \mathcal{S}$ should be bijective.

The mapping $f: \mathcal{S} \mapsto \mathcal{P}$ is called harmonic mapping, if $f$ satisfies

$$
\begin{aligned}
\Delta \xi(x, y, z) & =\xi_{x x}+\xi_{y y}+\xi_{z z}=0 \\
\Delta \eta(x, y, z) & =\eta_{x x}+\eta_{y y}+\eta_{z z}=0 \\
\Delta \zeta(x, y, z) & =\zeta_{x x}+\zeta_{y y}+\zeta_{z z}=0
\end{aligned}
$$


Different from the method in [31], in this paper, a new harmonic metric is proposed based on the variational formulation of the PDEs (13)(14)(15), which is the classical Dirichlet integral as follows,

$G=\int_{\Omega}(\nabla \xi)^{2}+(\nabla \eta)^{2}+(\nabla \zeta)^{2} d x d y d z$

The above function can be transformed from physical domain to the parametric domain using Jacobian transformation, which is denoted as variational harmonic metric as follows,

$$
\begin{aligned}
F_{\text {harmonic }} & =\int_{\mathcal{P}} \frac{\left[\frac{1}{3}\left(\left\|\boldsymbol{S}_{\xi}\right\|^{2}+\left\|\boldsymbol{S}_{\eta}\right\|^{2}+\left\|\boldsymbol{S}_{\eta}\right\|^{2}\right)\right]^{\frac{3}{2}}}{\operatorname{det} \mathbf{J}} d \mathcal{P} \\
& =\int_{\mathcal{P}} \frac{\left[\frac{1}{3} \operatorname{tr}\left(\mathbf{J}^{T} \mathbf{J}\right)\right]^{\frac{3}{2}}}{\operatorname{det} \mathbf{J}} d \xi d \eta d \zeta
\end{aligned}
$$

in which $\mathbf{J}$ is the Jacobian matrix of $\boldsymbol{S}(\xi, \eta, \zeta)$ as defined in Eqn. (9).

\subsubsection{Multi-objective optimization method for volumetric parameterization}

The orthogonality of iso-parametric structure is also a key quality measure of analysis-suitable volumetric parameterization in numerical simulation [35]. The orthogonality measure can be defined according to the differential geometry property of parametric volumes as follows ,

$F_{\text {orth }}=\int_{\mathcal{P}}\left\|\boldsymbol{S}_{\xi} \cdot \boldsymbol{S}_{\eta}\right\|^{2}+\left\|\boldsymbol{S}_{\eta} \cdot \boldsymbol{S}_{\zeta}\right\|^{2}+\left\|\boldsymbol{S}_{\xi} \cdot \boldsymbol{S}_{\zeta}\right\|^{2} d \mathcal{P}$

By combining the metrics defined in (11)(17)(18), a nonlinear optimization problem with multi-objective functions is achieved as follows

$\min _{C_{i, j, k}, \lambda_{i, j, k}}\left(1-\theta_{1}-\theta_{2}\right) F_{\text {harmonic }}+\theta_{1} F_{\text {unif }}+\theta_{2} F_{\text {orth }}$

where $\boldsymbol{C}_{i, j, k}$ and $\lambda_{i, j, k}$ are control points and weights as design variables to be solved, $\theta_{1}$ and $\theta_{2}$ are weights for the balance between the harmonic metric, uniformity metric and orthogonality metric.

Since the problem in (19) is usually a large-scale optimization problem, we adapt L-BFGS method to obtain the optimal solution, which is a quasi-Newton method for solving unconstrained nonlinear minimization problems. In L-BFGS framework, the inverse Hessian matrix of the objective function is approximated by a sequence of gradient vectors from previous iterations. For more details, the reader can refer to [22].

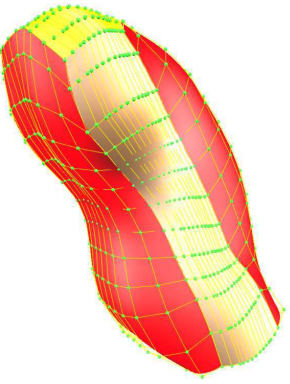

(a) Boundary NURBS surfaces

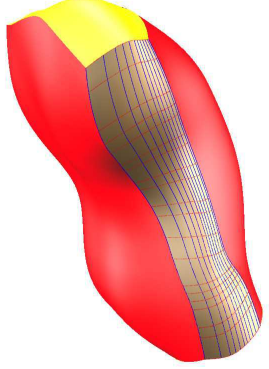

(c) Initial boundary parameterization

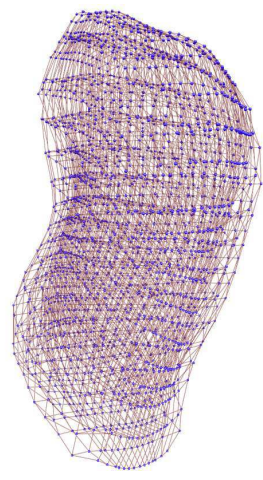

(e) Control lattice

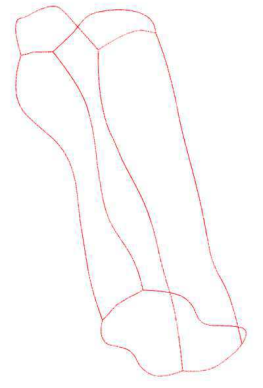

(b) Boundary NURBS curves

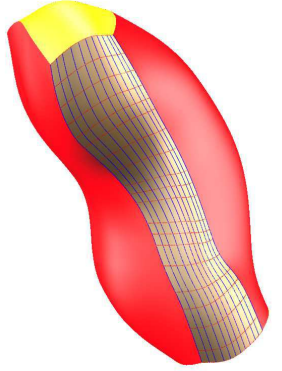

(d) Optimized boundary parameterization

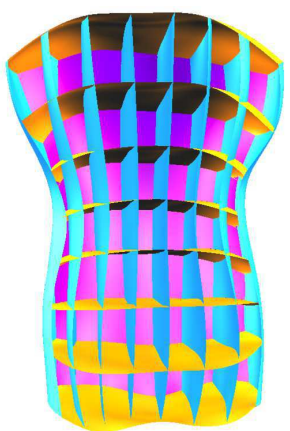

(f) Final isoparametric structure
Fig. 4 Volumetric parameterization of human body model.

\subsection{Experimental results}

Figure 4 shows an example for volumetric parameterization of human body. The given boundary NURBS surfaces and curves are shown in Figure 4 (a) and Figure 4 (b). Figure 4 (c) presents initial isoparametric net on one of the given boundary NURBS sufaces. Figure 4 (d) shows the uniformity-improved isoparametric net on the reparameterized boundary surface by optimal Möbius transformation. The control lattice of the final NURBS volumetric parameterization is shown in Figure 4 (e). To illustrate the quality of the parameterization, the iso-parametric surfaces of the resulting NURBS volume are presented in Figure 4 (f). More vol- 


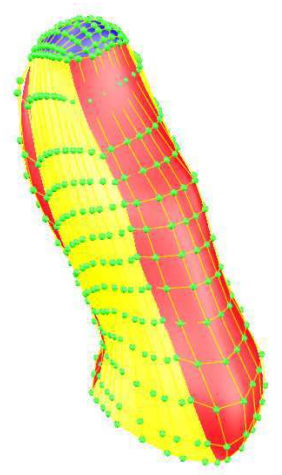

(a)

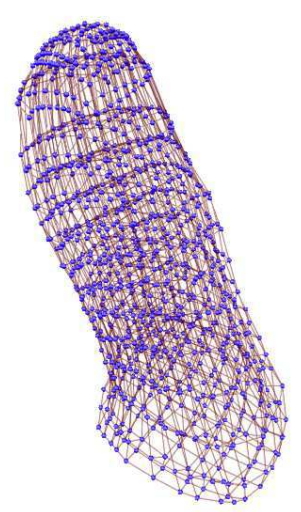

(c)

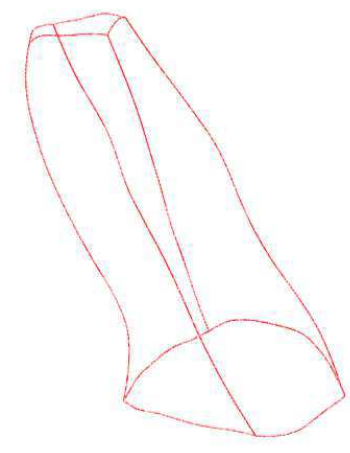

(b)

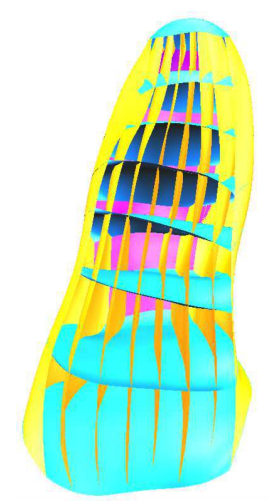

(d)
Fig. 5 Volumetric parametrization of thumb model : (a) boundary NURBS surfaces and control mesh; (b) boundary NURBS curves; (c)resulting control lattice; (d) final isoparametric structure.

Table 1 Quantitative data in Figure 2, Figure 4, Figure 5 and Figure 6. \# deg.: degree of B-spline parameterization; \# con.: number of control points; \# iter.: number of optimization iterations.

\begin{tabular}{cccc}
\hline Example & \# Deg. & \# Con. & \#Iter. \\
\hline Figure 2 & 4 & $8 \times 8 \times 8$ & 4 \\
Figure 4 & 3 & $13 \times 14 \times 13$ & 13 \\
Figure 5 & 3 & $8 \times 10 \times 13$ & 9 \\
Figure 6 & 3 & $8 \times 14 \times 11$ & 11 \\
\hline
\end{tabular}

umetric parameterization examples with complex geometry are shown in Figure 5 and Figure 6.

Quantitative data of the examples presented in Figure 2, Figure 4, Figure 5 and Figure 6 are summarized in Table 1. Overall, the volumetric parameterization obtained by the proposed two-stage method has highquality, and is suitable for isogeometric applications.

\section{Conclusion}

The quality of boundary parameterization has great effect on the subsequent volumetric parameterization results. Reparameterization methods can improve the quality of boundary parameterization without changing the geometry. In this paper, NURBS volumetric reparameterization is introduced into isogeometric analysis by using optimal Möbius transformation, and then the boundary surface reparameterization is performed as a pre-processing before constructing the inner control points and weights. Moreover, new uniformity metric and variational harmonic metric are also proposed for analysis-suitable volumetric parameterization. Experimental results illustrate that based on the reparameterization methods, we can obtain high-quality NURBS volumetric parameterization results, which are suitable for subsequent isogeometric analysis.

In the future, we will study the piece-wise reparameterization method for high-quality NURBS volumetric parameterization of computational domain [32]. The application of reparameterization technique in isogeometric solving on NURBS surfaces is also a part of our future work.

\section{Acknowledgment}

The first author is partially supported by the National Nature Science Foundation of China (Nos. 61004117, 61272390, 61211130103), the Defense Industrial Technology Development Program ( A3920110002), the Scientific Research Foundation for the Returned Overseas Chinese Scholars from State Education Ministry.

\section{References}

1. M. Aigner, C. Heinrich, B. Jüttler, E. Pilgerstorfer, B. Simeon and A.-V. Vuong. Swept volume parametrization for isogeometric analysis. In E. Hancock and R. Martin (eds.), The Mathematics of Surfaces (MoS XIII 2009), LNCS vol. 5654(2009), Springer, 19-44.

2. Y. Bazilevs, L. Beirao de Veiga, J.A. Cottrell, T.J.R. Hughes, and G. Sangalli. Isogeometric analysis: approximation, stability and error estimates for refined meshes. Mathematical Models and Methods in Applied Sciences, 6(2006) 1031-1090.

3. Y. Bazilevs, V.M. Calo, T.J.R. Hughes, and Y. Zhang. Isogeometric fluid structure interaction: Theory, algorithms, and computations. Computational Mechanics, 43(2008) 3-37.

4. Y. Bazilevs, V.M. Calo, J.A. Cottrell, J. Evans, T.J.R. Hughes, S. Lipton, M.A. Scott, and T.W. Sederberg. Isogeometric analysis using T-Splines. Computer Methods in Applied Mechanics and Engineering, 199(2010) 229263.

5. D. Burkhart, B. Hamann and G. Umlauf. Iso-geometric analysis based on Catmull-Clark subdivision solids. Computer Graphics Forum, 29(2010) 1575-1584.

6. E. Cohen, T. Martin, R.M. Kirby, T. Lyche and R.F. Riesenfeld, Analysis-aware Modeling: Understanding Quality Considerations in Modeling for Isogeometric Analysis. Computer Methods in Applied Mechanics and Engineering, 199(2010) 334-356. 


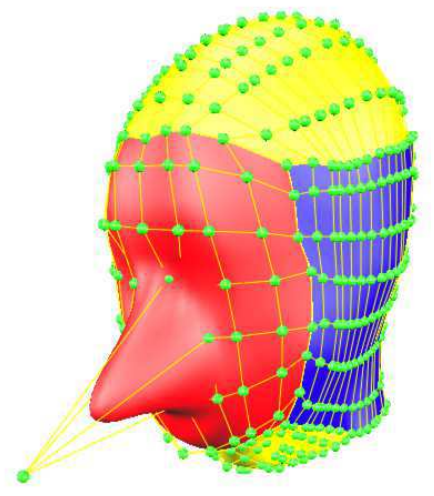

(a) Boundary NURBS surfaces

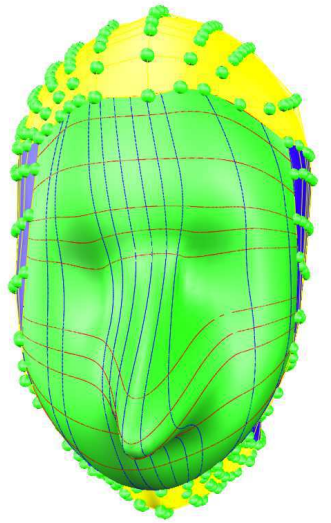

(b) Initial boundary parameterization

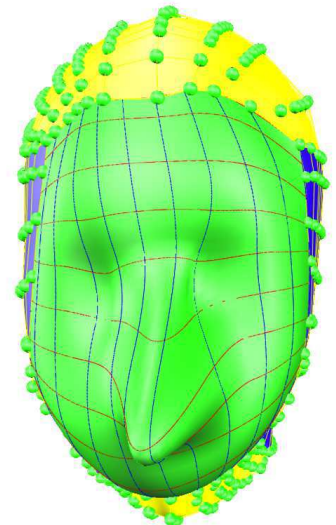

(c) Optimized boundary parameterization

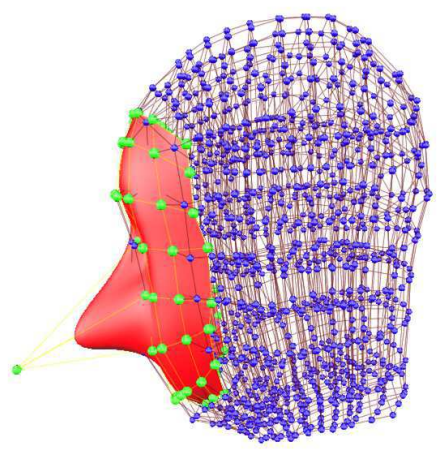

(d) Control lattice

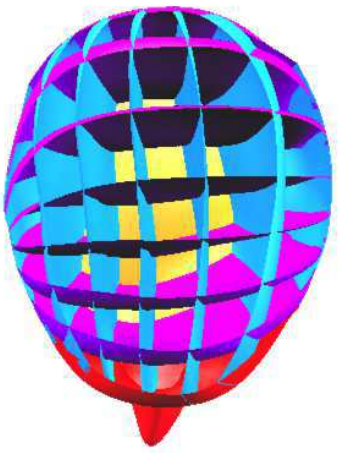

(e) Final isoparametric structure (top view)

Fig. 6 Volumetric parameterization of head model.

7. J.A. Cottrell, T.J.R. Hughes, Y. Bazilevs. Isogeometric Analysis: Toward Integration of CAD and FEA. Wiley, 2009

8. J.A. Cottrell, T.J.R. Hughes, and A. Reali. Studies of refinement and continuity in isogeometric analysis. Computer Methods in Applied Mechanics and Engineering, 196(2007) 4160-4183.

9. J.A. Cottrell, A. Reali, Y. Bazilevs, and T.J.R. Hughes. Isogeometric analysis of structural vibrations. Computer Methods in Applied Mechanics and Engineering, 195(2006) 5257-5296.

10. M. Dörfel, B. Jüttler, and B. Simeon. Adaptive isogeometric analysis by local h-refinement with T-splines. Computer Methods in Applied Mechanics and Engineering, 199(2010) 264-275.

11. G. Elber, Y. Kim, M. Kim. Volumetric boolean sum. Computer Aided Geometric Design, 29(7): 532-540, 2012.

12. J.M. Escobara, J.M. Cascónb, E. Rodrígueza, R. Montenegro. A new approach to solid modeling with trivariate T-spline based on mesh optimization. Computer Methods in Applied Mechanics and Engineering, 200(2011) 32103222 .

13. H. Gomez, V.M. Calo, Y. Bazilevs, and T.J.R. Hughes. Isogeometric analysis of the Cahn-Hilliard phase-field model. Computer Methods in Applied Mechanics and Engineering, 197(2008) 4333-4352.

14. T.J.R. Hughes, J.A. Cottrell, Y. Bazilevs. Isogeometric analysis: CAD, finite elements, NURBS, exact geometry, and mesh refinement. Computer Methods in Applied Mechanics and Engineering, 194(2005) 4135-4195.

15. Y. Jia, Y. Zhang, G. Xu, X. Zhuang, T. Rabczuk. Reproducing kernel triangular B-spline-based FEM for solving PDEs. Computer Methods in Applied Mechanics and Engineering, 267(2013) 342-358.

16. Lee, E. and Lucian, M. Möbius reparameterization of rational B-splines. Computer Aided Geometric Design, 8(1991): $213-238$.

17. L. Liu, Y. Zhang, T. J.R. Hughes, M. A. Scott, T. W. Sederberg. Volumetric T-Spline construction Using Boolean operations. Engineering with Computers, 2014. DOI: $10.1007 / \mathrm{s} 00366-013-0346-6$

18. T. Martin, E. Cohen, and R.M. Kirby. Volumetric parameterization and trivariate B-spline fitting using harmonic functions. Computer Aided Geometric Design, 26(2009):648-664.

19. Nguyen-Thanh N., Kiendl J., Nguyen-Xuan H., Wuchner R., Bletzinger K.U., Bazilevs Y., Rabczuk T.. Rotation free isogeometric thin shell analysis using PHT-splines. Computer Methods in Applied Mechanics and Engineering, 2011, 200(47-48), 3410-3424

20. T. Nguyen, B. Jüttler. Using approximate implicitization for domain parameterization in isogeometric analysis. International Conference on Curves and Surfaces, Avignon, France, 2010.

21. N. Nguyen-Thanh, H. Nguyen-Xuan, S.P.A. Bordasd and T. Rabczuk. Isogeometric analysis using polynomial 
splines over hierarchical T-meshes for two-dimensional elastic solids. Computer Methods in Applied Mechanics and Engineering, 200(2011) 1892-1908.

22. J. Nocedal and S. J. Wright. Numerical optimization. Springer verlag, 1999.

23. E. Pilgerstorfer, B. Jüttler . Bounding the influence of domain parameterization and knot spacing on numerical stability in Isogeometric Analysis. Computer Methods in Applied Mechanics and Engineering, 268(2014), 589-613

24. K.F. Pettersen, V. Skytt. Spline volume fairing. 7th International Conference on Curves and Surfaces. Avignon, France, June 24-30, 2010, Lecture Notes in Computer Science, 6920(2012) 553-561.

25. C.L. Wang, K. Tang. Non-self-overlapping Hermite interpolation mapping: a practical solution for structured quadrilateral meshing. Computer-Aided Design, 37(2005) 271-283.

26. W. Wang, Y. Zhang, L. Liu, T. J.R. Hughes. Trivariate solid T-spline construction from boundary triangulations with arbitrary genus topology. Computer-Aided Design, 45(2013) 351-360.

27. X. Wang, X. Qian. An optimization approach for constructing trivariate B-spline solids. Computer-Aided Design, 46(2014) 179-191.

28. G. Xu, B. Mourrain, R. Duvigneau, A. Galligo. Optimal analysis-aware parameterization of computational domain in 3D isogeometric analysis. Computer-Aided Design, 45(2013), 812-821.

29. G. Xu, B. Mourrain, R. Duvigneau, A. Galligo. Parameterization of computational domain in isogeometric analysis: methods and comparison. Computer Methods in Applied Mechanics and Engineering, 200(2011), 2021-2031.

30. G. Xu, B. Mourrain, R. Duvigneau, A. Galligo. Analysissuitable volume parameterization of multi-block computational domain in isogeometric applications. ComputerAided Design, 45(2013), 395-404.

31. G. Xu, B. Mourrain, R. Duvigneau, A. Galligo. Constructing analysis-suitable parameterization of computational domain from CAD boundary by variational harmonic method. Journal of Computational Physics , 2013, 252, 275-289

32. J. Yang, D. Wang, H. Hong. Improving angular speed uniformity by $C^{1}$ piecewise reparameterization. Automated Deduction in Geometry 2012: 33-47

33. Y. Zhang, W. Wang, T. J.R. Hughes. Solid T-spline construction from boundary representations for genus-zero geometry. Computer Methods in Applied Mechanics and Engineering, 201(2012) 185-197.

34. Y. Zhang, W. Wang, T. J.R. Hughes. Conformal solid T-spline construction from boundary T-spline representations. Computational Mechanics, 51(2013) 1051-1059.

35. Y. Zhang, Y. Jia, S.Y. Wang. An improved nearlyorthogonal structured mesh generation system with smoothness control functions. Journal of Computational Physics, 231(2012) 5289-5305. 